\title{
GLOBAL SOLUTIONS TO THE NONLINEAR HYPERBOLIC PROBLEM
}

\author{
Nikolai A. Larkin \\ The Institute of Theorical and Applied Mechanics, Novosibirsk-90, 630090, Rusia*
}

\section{ABSTRACT}

We prove the existence and uniqueness of global regular solutions to the mixed problem for the nonlinear hyperbolic equation with nonlinear damping.

$$
\begin{gathered}
u_{t t}-a(u) u+\left|u_{t}\right|^{\rho} u_{t}=f(x, t) \text { in }(0,1) x(0, T)=Q, \\
u(0, t)=u(1, t)=0, \\
u(x, 0)=u_{0}(x), \quad u_{t}(x, 0)=u_{1}(x)
\end{gathered}
$$

Where $a(u) \geq a_{0}>0, \rho>1$. No restrictions on a size of $u_{0}, u_{1}, f$ are imposed.

It is well-known that quasilinear hyperbolic equations, generally speaking, do not have regular solutions for all $t>0$. Their solutions can blow up at a finite period of time. See examples of such singularities in $[1,3]$. On the other hand,it was observed that adding a linear damping to the nonlinear hyperbolic equations one can expect the existence of global regular solutions provided initial conditions and right-hand side have sufficiently small appropriate norms, [2]. Moreover, in [3] was shown that the presence of the nonlinear damping allows to prove the existence of regular solutions for the equation

$$
K(u) u_{t t}-\Delta u+\left|u_{t t}\right|^{\rho} u_{t t}=f, K>0 .
$$

without restrictions on a size of the initial data and $f$.

Later, using the idea of [3], we proved in [4] the existence of regular solutions for the damped Carrier equation.

$$
u_{t t}-M\left(|u(t)|^{2}\right) \Delta u+\left.\alpha \cdot u_{t}\right|^{\rho} u_{t}=f
$$

without smallness conditions for the initial data and $f$.

\footnotetext{
* Supported by CNP-Brazil as Visiting Profesor as the State University of Maringá.
} 
Here, we continue to exploit this idea and consider the following nonlinear mixed problem.

$$
\begin{gathered}
u_{t t}-a(u) u+\left|u_{t}\right|^{\rho} u_{t}=f(x, t) \text { in } Q=(0,1) x(0, T), \\
u(0, t)=u(1, t)=0 \\
u(x, 0)=u_{0}(x), \quad u_{t}(x, 0)=u_{1}(x)
\end{gathered}
$$

where $a(u)$ is a smooth positive function.

Unlike the Carrier equations. (1) has local nonlinearities: the function $a(u)$ depends on a solution; and the function $M\left(|u(t)|^{2}\right.$ depends on the $L_{2}$ - norm of it. This difference makes study of (1)-(3) more complicated and forces us to consider only the onedimensional case. Nevertheless, the basic technique is similar to one used in the case of the Carrier equation in [4]. Under natural conditions for $a(u)$, we prove the existence and uniqueness of regular solutions to (1)-(3) without any restrictions on a size of $u_{0}, u_{1}, f$.

\section{Assumptions.}

A1. $a(u) \in C^{1}(R) ; a(u) \geq a_{0}>0$.

A2. $a_{u} \leq \operatorname{Aa}(u)$

A3. $1<\rho$,

Where $a_{0}, A$ are positive constants.

In the sequel, we use standard notations for functional spaces, see [5].

Theorem. Let $\mathrm{T}$ be an arbitrary positive number; $u_{0} \in H^{2}(0,1) \cap H_{0}^{1}(0,1)$ and A1-A3 hold. The for any f such that $f, f_{t} \in L^{2}$, there exists a unique regular solution to (1)-(3), $u(x, t)$ :

$$
\begin{gathered}
u \in L^{\infty}\left(0, T ; H^{2}(0,1) \cap H_{0}^{1}(0,1)\right), \\
u_{t} \in L^{\infty}\left(0, \check{T} ; H_{0}^{1}(0,1)\right), \\
u_{t t} \in L^{\infty}\left(0, T ; L^{2}(0,1)\right)
\end{gathered}
$$




\section{The scheme of the proof.}

The assumption A1 allows to rewrite (1) in the equivalent form

$$
\frac{1}{a(u)} u_{t}-u+\frac{\left|u_{t}\right|^{\rho} u_{t}}{a(u)}=\frac{f}{a(u)}
$$

Obviously, solutions to (4), (2), (3) are also solutions to (1)-(3). Equation (4) is similar to the class of quasilinear hyperbolic equations studied in [4] with exception of the damping that can degenerate when $a(u) \rightarrow \infty$. Also, the coefficient of $u_{t t},\left(\frac{1}{a(u)}\right)$ can be zero. It means that (4) is the degenerated hyperbolic equation. Morever, dependence of the damping term of $u$ and $u_{t}$ brings more difficulties to analysis of (4), (2), (3). Nevertheless, we can employ in our case the technique developed in [4].

Approximate solutions to (4), (2), (3) will be constructed by the Faedo-Galerkin method with the special basis. Let $w_{j}(x)$ be eigen-functions of the problem

$$
\begin{gathered}
w_{j x x}+\lambda_{j} w_{j}=0 \text { in }(0,1), \\
w_{j}(0)=w_{j}(1)=0 .
\end{gathered}
$$

Then for $\in>0$

$$
u_{\epsilon}^{N}(x, t)=\sum_{j=i}^{N} g_{j}^{N}(t) w_{j}(x)
$$

where unknown functions $g_{j}(t)$ are solutions to the following Cauchy problem

$$
\left(\left(\in+\frac{1}{a\left(u_{\epsilon}^{N}\right)}\right) u_{\in t t}^{N}, w_{j}\right)(t)+\left(u_{\in x}^{N}, w_{j}\right)(t)+\left(\frac{u_{\epsilon t}^{N}{ }^{\rho} u_{\epsilon t}^{N}}{a\left(u_{\epsilon}^{N}\right)}, w_{j}\right)(t)=\left(\frac{f}{a\left(u_{\epsilon}^{N}\right)}, w_{j}\right)(t)
$$




$$
\begin{aligned}
& g_{j}^{N}(0)=\alpha_{j}=\left(u_{0}, w_{j}\right), \\
& g_{j t}^{N}(0)=\beta_{j}=\left(u_{1}, w_{j}\right), j=1, \ldots ., N .
\end{aligned}
$$

Here $(u, v)(t)=\int_{0}^{1} u(x, t) v(x, t) d x$.

The system of nonlinear ordinary differential equations (6) is not solved with respect to $g_{j t t}$, but it can be transformed a normal system of ODE due to the fact that the matrix $\in I+\left(\sum \frac{1}{a\left(u \in \in^{N}\right)} w_{j}, w_{i}\right), i, j=1, \ldots, N$, is positive for $\in>0$, see A1.

Hence, the Cauchy problem (6), (7) has solutions $g_{j}^{N}$ at some interval $\left(0, T_{N}\right)$, and we need a priori estimates in order to prolongate solutions to the interval $(0, T)$ and to pass to the limits when $\in \rightarrow 0$ and $N \rightarrow \infty$.

\section{The First Estimate}

Multiplying (6) by $g_{j t}^{N}$ and using A1-A3, after some calculations we come to the inequality

$$
\begin{aligned}
& \int_{0}^{1}\left(\frac{\left|u_{\in t}^{N}(x, t)\right|^{2}}{a\left(u_{\in}^{N}\right)}+\left|u_{\in x}^{N}(x, t)\right|^{2}\right) d x+\int_{0}^{t} \int_{0}^{1} \frac{\left|u_{\in t}^{N}\right|^{\rho+2}}{a\left(u_{\epsilon}^{N}\right)} d x d r \leq \\
& C_{l}\left(\left\|u_{0}\right\| H_{0}^{l}(0,1),\left\|u_{l}\right\| L^{3}(0,1),\|f\| L^{3}(Q)\right),
\end{aligned}
$$

where $\mathrm{C}$ does not depend on $\in, N, t$.

From here and from A1.

$$
\begin{aligned}
& \text { Sup } \max \mid u(x, t) \leq C_{2} . \\
& t \in(0, T)^{x \in(0,1)}
\end{aligned}
$$


This imply

$$
a_{0} \leq a(u) \leq M<\infty
$$

Where $C_{2}, M$ do not depend on $\in, N, T$.

\section{The Second Estimate}

Taking the derivate of (6) with respect to $t$, multiplying the result by $g_{j t t}^{N}$, after standard transformations we obtain

$$
\begin{aligned}
& \int_{0}^{1}\left(\left|u_{\in t t}^{N}(x, t)^{2}+\right| u_{\in x t}^{N}(x, t)^{2}\right) d x \leq \\
& C_{3}\left(\left\|u_{0}\right\|_{H^{2}}(0,1) \cap H_{0}^{l}(0,1),\left\|u_{1}\right\|_{H_{0}^{l}}^{l}(0,1),\|f\|_{H^{l}}\left(0, T, L^{3}(0,1)\right)\right),
\end{aligned}
$$

where $\mathrm{C}_{3}$ does not depend on $\in, N, t$.

Finally, taking into account (5) and estimates (8)-(11), we get

$$
\int_{0}^{1}\left|u_{\in x x} \quad(x, t)\right|^{2} d x \leq C_{4}
$$

With (8)-(12) it is easy to pass to the limits in (6) when $N \rightarrow \infty, \in \rightarrow 0$, hence, to prove the existence of regular solutions to (4), (2), (3) and, consequently, to (1)-(3). Uniqueness may be proved in the usual way.

Theorem is proved.

Remark. The function $a(u)$ can depend on $x, t$. 


\section{REFERENCES}

1. ROZHDESTVENSKII, B.L., YANENKO, N. N., Systems of quasilinear equations and their applications to gas dynamics, Providence, AMS, (1983).

2. MATSUMURA, A., Global existence and asymptotics of the solutions of the second-order quasilinear hyperbolic equations with the first-order dissipation, Publ. Res. Inst. Sci., Kyoto Univ., 13, P. 349-379 (1977).

3. LARKIN, N.A., Global solvability of boundary value problems for a class of quasilinear hyperbolic equations, Siber. Math. Journal, v.22, N1, p. 82-88 (1981).

4. FROTA, C., LARKIN, N.A., On local and global solvability of nonlinear mixed problems modeling vibrations of string, Proc. Of 7-th Intern. Col. on Dif. Equats., August (1996), Plovdiv, Bulgaria, VPS Edition, p. 381-395 (1997).

5. LIONS, J.-L., MAGENES, E., Problemes aux limites non homogenes, Aplications, Dunod, Paris, v.1, (1968). 